\title{
ESTIMATED PREVALENCE OF JOINT HYPERMOBILITY SYNDROME IN PATIENTS OF A HOSPITAL IN RECIFE-PE
}

Pedro Falcão de Melo Cavalcanti', Marcus Villander Barros de Oliveira Sá1,2,3,*, Gustavo Henrique de Sá Miranda Cavalcante Filho', Flávio José Siqueira Pacheco', Jorge Luiz Carvalho Figueredo', Luydson Richardson Silva Vasconcelos ${ }^{2,3}$, Francisco José Trindade Barretto ${ }^{1}$

1.Real Hospital Português de Beneficência em Pernambuco, Recife (PE), Brazil. 2.Fundação Oswaldo Cruz, Recife (PE), Brazil. 3.Universidade de Pernambuco, Recife (PE), Brazil.

*Corresponding author: marcusvillander@yahoo.com.br

\section{BACKGROUND}

Ehlers-Danlos syndrome (EDS) is a relatively rare hereditary disorder of connective tissue whose genetic alterations lead to the loss of collagen synthesis and to the appearance of joint hypermobility, skin hyperextensibility and tissue fragility. It is currently known that there are 13 clinical syndromes associated with EDS and frequently patients with hypermobile subtype (hEDS) or joint hypermobility syndrome (JHS) seek medical care for musculoskeletal pain of varying intensity, with no apparent cause and that compromise their quality of life. Chronic pains can trigger social isolation, anxiety, depression and disability. Ehlers-Danlos syndrome is a neglected disease and its subtypes are no properly evaluated and documented. Its prevalence in Brazilian population is unknown and the lack of data contributes to perpetuate the lack of knowledge and diagnosis.

\section{MATERIALS AND METHODS}

A pilot cross-sectional study to determine the estimated prevalence of JHS in patients hospitalized in a private hospital in Recife-PE, in the period between August and September 2018.The Beighton score was used to determine the presence of joint hypermobility and the Brighton criteria was used for the diagnosis of JHS. Inclusion criteria: age between 15 and 50 years, patients of both genders and in hospital for at least 48 hours. Patients with some physical limitation or motor disability, those who were in intensive care unit (ICU), bedridden or individuals with cognitive impairment that limited the evaluation were excluded from the study.

\section{RESULTS}

Fifty patients were examined, 12 were excluded because they did not meet the eligibility criteria. The average age of the individuals was 32.9 years and $76.3 \%$ (29) were female. Seventeen of 38 patients fulfilled criteria for joint hypermobility (Beighton score); 8 patients fulfilled the Brighton criteria for JHS, estimated prevalence of $21 \%$. Of those 8 patients with JHS, 4 suffered from joint pain and 3 had depression and anxiety. Only 1 of the JHS (1/8) patients were aware of the diagnosis prior to the study.

\section{CONCLUSION}

Joint hypermobility syndrome is a disease found in clinical practice, but not routinely recognized during clinical examination. In this small pilot study, the estimated prevalence of JHS is considered high. Larger research should be carried out to establish the prevalence of JHS in each location, in order to disseminate knowledge and lead to the identification and correct management of these patients. 